\title{
Evidence-based medicine: A resource for all
}

\author{
LR Sutherland MD FACP FRCPC
}

$\mathrm{N}$ early a decade has passed since the announcement of a paradigm shift by the Evidence-Based Medicine Working group (1). No longer would decisions be made based on content expertise and clinical experience. Future decision making would rest on the complete identification of original studies and application of the tools of critical appraisal. The concept swept the world and was avidly taken up by many. A cursory search of MEDLINE revealed two citations in 1992 and 1082 in 1999; in 2001, 1492 citations are already available. New journals have been published, and new textbooks written.

Evidence-based medicine is nothing without the evidence on which objective decision making can occur. In many areas, the Cochrane Collaboration plays a leading role in the assembly and critique of the available evidence. The headquarters of the Collaboration is based in Oxford, but Cochrane Centres, including one at McMaster University, Hamilton, Ontario, are located around the world. From a gastroenterological and hepatological perspective, the relevant review groups are inflammatory bowel disease and irritable bowel syndrome (chaired by J McDonald, London, Ontario), hepatobiliary disease (Christiann Gluud, Copenhagen, Denmark), upper gastointestinal and pancreatic disease (David Forman, Leeds, United Kingdom), and colorectal cancer (Peer Willie-Jorgensen, Copenhagen, Denmark).

What has been accomplished to date is a project that "rivals the human genome project in its complexity" (2). One of the first challenges was to identify all of the previously published clinical trials that had not always been cor- rectly identified in MEDLINE. The hand-searching of the published literature to identify all of the clinical trials is well underway. Search strategies for the identification of clinical trials have been published (3). The Cochrane Library is published quarterly and is so large that it requires two compact discs to be distributed.

A systematic review differs from a narrative review in several key areas. A protocol is first developed to identify the research question, determine the inclusion criteria for trials, construct a measure of quality and define a search strategy for identifying the relevant literature. After the first database search, additional trials are often identified by review of the bibliographies of the articles identified in the first search. Data are extracted, and if sufficient numbers of patients or trials are identified, a statistical analysis or metaanalysis may be performed.

In this issue, McDonald and colleagues (pages 17-21) report that clinicians are taking up the message of looking to systematic reviews for guidance rather than the narrative review of the past. In their study, participants were randomly provided with either a systematic review or a narrative review regarding the use of azathioprine in the treatment of Crohn's disease. A series of questions probed the ability of either review to change their pattern of practice. For most participants, the systematic review was more compelling in encouraging a change in practice pattern.

What challenges lie head? The Cochrane Collaboration is underfunded in many countries, including Canada. This year, the provincial Ministers of Health provided only $\$ 100,000$ to be distributed across the 16 medical faculties in

University of Calgary, Calgary, Alberta

Correspondence: Dr LR Sutherland, Department of Community Health Sciences, Heritage Medical Research Building, University of Calgary, 3330 Hospital Drive NW, Calgary, Alberta T2N 4N1. Telephone 403-220-7181, fax 403-270-7307, e-mail lsutherl@ucalgary.ca 
Canada. The Canadian Institutes for Health Research is considering additional support. Most of the work is done on a voluntary basis, and volunteers tend to burn out over time. The commitment to update each review as soon as new studies are published is commendable, but some reviewers lose interest over time. While the first review may be copublished in a peer-reviewed journal, there is little interest in publishing the 10th revision.

In many cases, the evidence required for decision making may not yet be available. The choice of review topic tends to be driven by curiousity and may reflect the availability of resources. Taking on a systematic review of Helicobacter pylori treatment would be a daunting task, with at least 100 trials to be reviewed and analyzed. In contrast, when reviewing azathioprine in the induction of remission of ulcerative colitis, there are insufficient trials to reliably inform the decision maker.

What can we do? Those of us who are academic leaders need to increase the awareness of our colleagues at the annual review, and of promotion and tenure committees, that this is important work that needs to be recognized. We need to talk about the importance of evidence-based medicine and the Cochrane Collaboration. We need to encour- age our hospital and university libraries to carry The Cochrane Library, which should also be readily accessible to everyone. National or disease-specific registries of clinical trials need to be in place to faciliate future reviews. New techniques to identify publication bias need to be developed (4). We need to consider the mosaic of evidence that not only includes well designed clinical trials but also provides for input from well designed cohort or case control studies.

In the current environment, with concerns about the sustainability of the health care system, evidence-based medicine has an important role to play. Along with technology assessment units, it has the potential to help us rationalize care for the benefit of our patients rather than ration it for future generations.

\section{REFERENCES}

1. Evidence-Based Medicine Working Group. Evidence-Based Medicine A new approach to teaching the practice of medicine. JAMA 1992;268:2420-5.

2. Naylor CD. Grey zones of clinical practice: some limits to evidencebased medicine. Lancet 1995;345:840-2.

3. Chalmers I, Altman D, eds. Systematic Reviews. London: BMJ Publishing Group, 1995.

4. Shaheen NJ, Crosby MA, Bozymski EM, Sandler RS. Is there publication bias in the reporting of cancer risk in Barrett's esophagus. Gastroenterology 2000;119:333-8. 


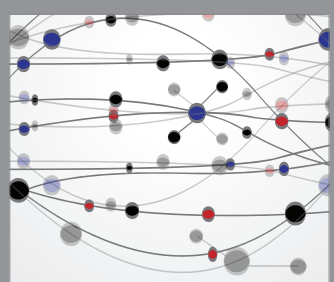

The Scientific World Journal
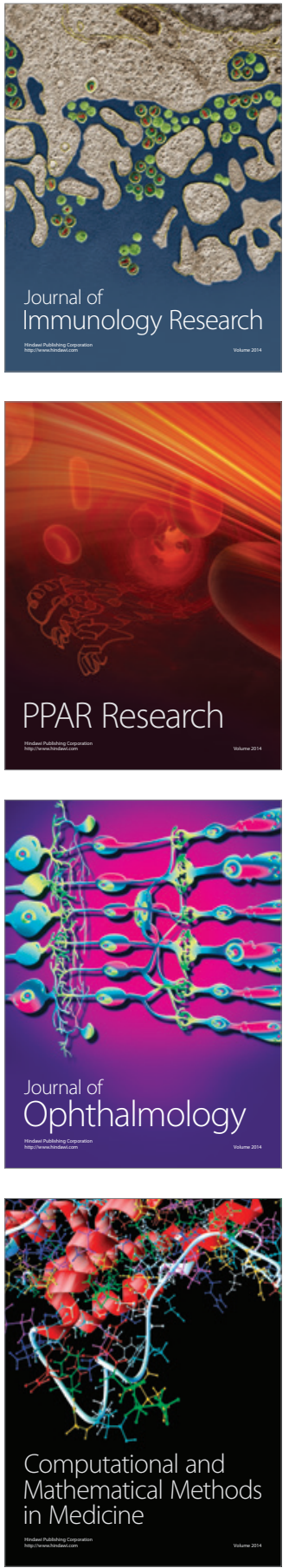

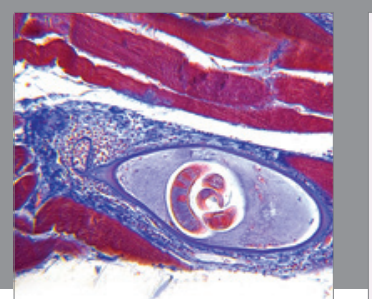

Gastroenterology Research and Practice

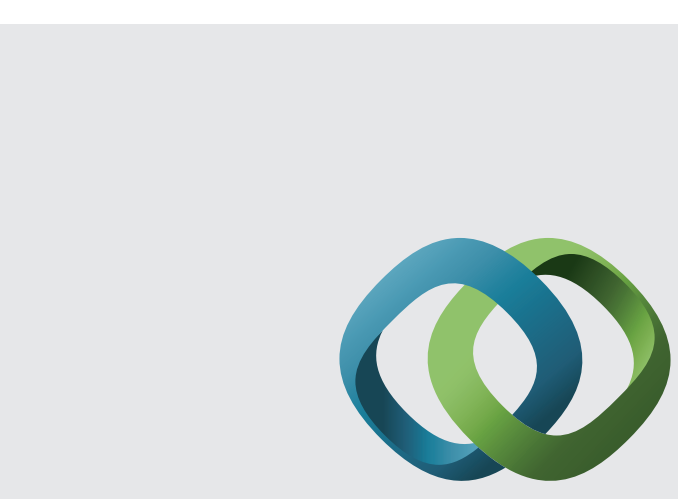

\section{Hindawi}

Submit your manuscripts at

http://www.hindawi.com
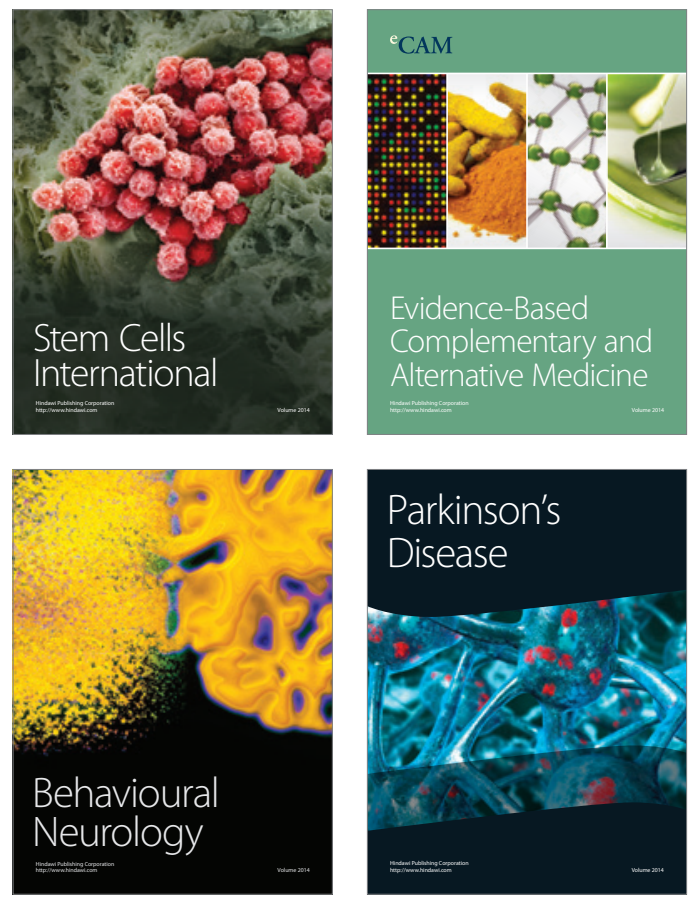
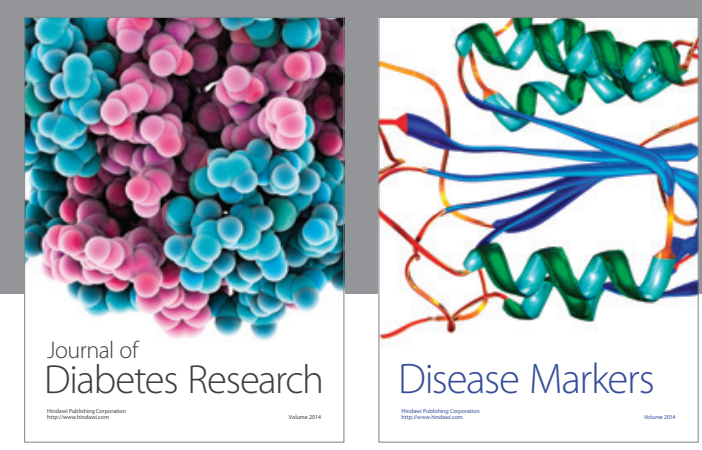

Disease Markers
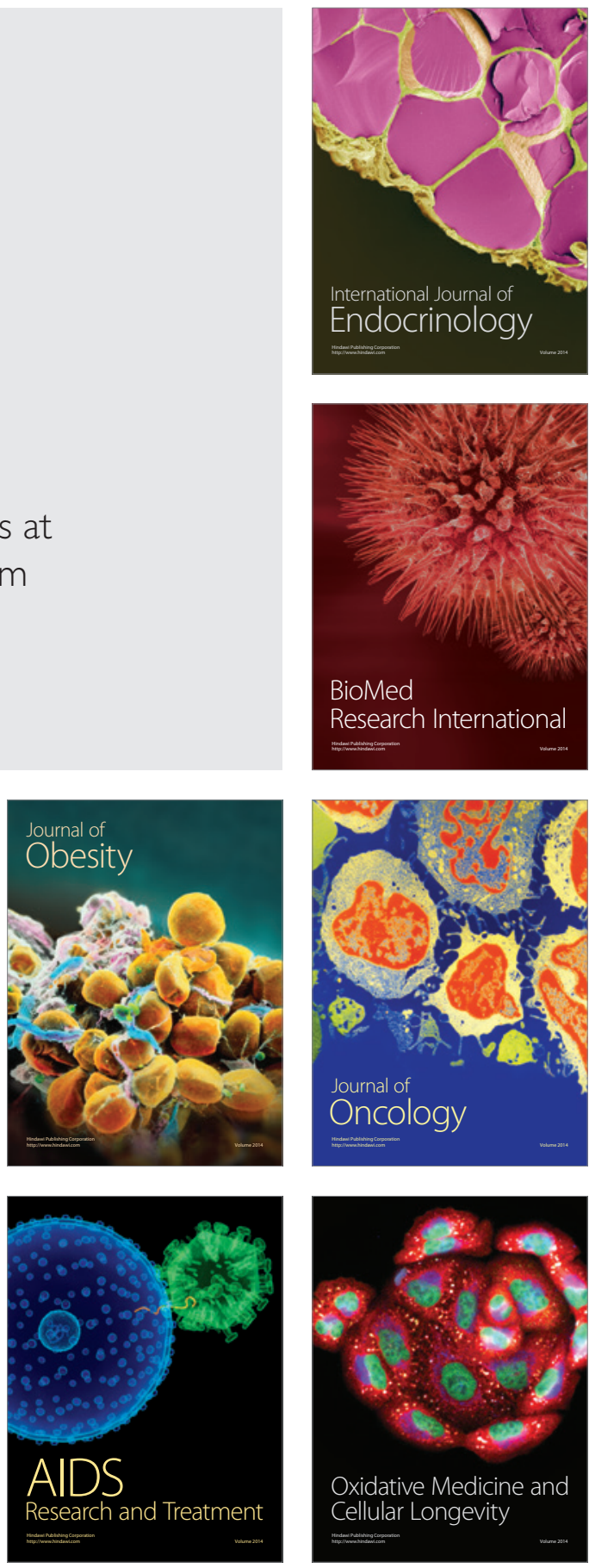